\title{
Hardware-software system for simulating and analyzing earthquakes applied to civil structures
}

\author{
J. P. Amezquita-Sanchez, R. A. Osornio-Rios, R. J. Romero-Troncoso, and A. Dominguez-Gonzalez \\ HSPdigital Research Group, C. A. Mecatrónica, Facultad de Ingeniería, Campus San Juan del Río, \\ Universidad Autónoma de Querétaro, Moctezuma 249, Col. San Cayetano, 76807 San Juan del Río, Querétaro, México
}

Correspondence to: R. A. Osornio-Rios (raosornio@ hspdigital.org)

Received: 11 October 2011 - Revised: 17 November 2011 - Accepted: 27 November 2011 - Published: 6 January 2012

\begin{abstract}
The occurrence of recent strong earthquakes, the incessant worldwide movements of tectonic plates and the continuous ambient vibrations caused by traffic and wind have increased the interest of researchers in improving the capacity of energy dissipation to avoid damages to civil structures. Experimental testing of structural systems is essential for the understanding of physical behaviors and the building of appropriate analytic models in order to expose difficulties that may not have been considered in analytical studies. This paper presents a hardware-software system for exciting, monitoring and analyzing simultaneously a structure under earthquake signals and other types of signals in real-time. Effectiveness of the proposed system has been validated by experimental case studies and has been found to be a useful tool in the analysis of earthquake effects on structures.
\end{abstract}

\section{Introduction}

Recent strong earthquakes (e.g. Japan, Chile, and México), the incessant worldwide movements of tectonic plates and the continuous ambient vibrations caused by traffic and wind have increased the interest of researchers in improving the capacity of energy dissipation to avoid damages to civil structures (Dyke, 1998). In order to achieve this task, it is necessary to perform the simulation of structures under vibration loads. This is usually performed either experimentally or analytically. Experimental testing of structural systems is essential to understand physical behaviors and build appropriate analytic models in order to expose difficulties that may not have been considered in analytical studies (Jeyasehara et al., 2009).

Recently, structural simulators have gained importance, particularly in countries vulnerable to destructive earthquakes. A structural simulator is defined as a system capable of providing the most direct experimental pattern for the performance assessment of structures subject to earthquakes or to any ground motion (Nakata, 2010). The general block diagram of a structural simulator is shown in Fig. 1. The exciter, typically a shaker, actuates on the structure, producing vibrations which are measured by sensors - generally accelerometers. Then, the monitored signal is analyzed by means of processing techniques such as FFT, wavelet, and others, in order to get the response of the structure in detail.

In order to excite a structure, several kinds of signals have been used as inputs for the shaker such as: recorded earthquakes, synthetic signals, and ambient vibrations. Different works have proposed the use of earthquake signals to excite civil structures, due to the fact that it permits analysis of the structure behavior under real conditions (San Bartolome et al., 2004; Dolce and Cardone, 2006; Johnson et al., 2008; Van de Lindt and Potts, 2008; Kamanli and Balik, 2010). Synthetic signals are mainly used to obtain the modal parameters of a structure and to determine its fatigue life; sinusoidal sweep or pure sinusoidal are the most widely used (Zivanović et al., 2006; Dominguez et al., 2007; Ibrahim and Reynolds, 2008; Ersubasi and Korkmaz, 2010). Another kind of signal frequently used to excite a structure is ambient vibration such as traffic and wind. As well as synthetic signals, ambient vibration is used to obtain the modal parameters of a structure (Brownjohn et al., 2003; Ren et al., 2004). From the above stated, it is necessary to have a simulator capable of synthesizing or generating the three types of signals, so as to excite any kind of structure under different conditions.

Regarding structure sensing, current literature points out that accelerometers (Ren et al., 2004; Dolce and Cardone, 2006; Zivanović et al., 2006; Ibrahim and Reynolds, 2008) and string potentiometers (Van de Lindt et al., 2008) are two 
Structural Simulator

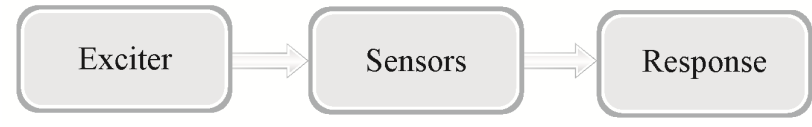

Fig. 1. Structural simulator.

of the most widely used sensors for monitoring a structure during an excitation. An existing problem in several works is the quantity of test points that are simultaneously monitored. For instance, Zivanović et al., 2006 proposed the monitoring of a lively steel box girder footbridge. The structure was tested in several points; however, the monitoring of the structural response was only done by one accelerometer. This is considered unsuitable to know the behavior of the structure in detail. Conversely, aiming at observing the behavior of the structure in detail, simultaneous monitoring of several points is necessary. Therefore, the development of a system capable of monitoring concurrently several test points is considered essential.

Concerning the signal processing techniques used for analyzing the response of a structure, several works are based on the fast Fourier transform (FFT) (Chen et al., 2005; Dolce and Cardone, 2006; Dominguez et al., 2007); the short time Fourier transform (STFT) (Dolce and Cardone, 2006; Nagarajaiah, 2009; Nagarajaiah and Basu, 2009); discrete wavelet transform (DWT) (Ovanesova and Suárez, 2004), and the wavelet packet transform (WPT) (Sun and Chang, 2002; Han et al., 2005). Nevertheless, all these works present several disadvantages. First, they use two software programs, one for generating the signal that excites the structure and another for the analysis of the measured signal, which limits the software portability and the comparison between both signals. In addition, the lack of online processing and portability of their systems is also observed. For these reasons, it would be desirable to have a versatile and portable system capable of simultaneously generating, modifying, reproducing, and analyzing any kind of signal so as to be used in real-time structural analysis.

The contribution of this work is the design, development and implementation of a hardware-software system for exciting, monitoring, and analyzing civil structures simultaneously. The proposed system is capable of synthesizing, generating, modifying and controlling the three most common used signals such as earthquakes, ambient vibrations and synthetic signals to excite a structure. Moreover, the system has the ability to adapt the monitored signal to most of the commercial electrodynamic shakers. Additionally, the system has the potential for continuous and online monitoring to observe the real structure performance during an earthquake or any other excitation, as well as the signal analysis in one or several test points, concurrently. Another contribution of this work is that the software is capable of analyzing the measured signals through several techniques such as FFT, STFT, DWT, WPT, and their combination. The effectiveness of the proposed system is validated through different experimental cases, where earthquake signals, traffic effects, and synthetic signals are used to stimulate a truss-type scaled structure.

\section{Theoretical background}

This section presents the theoretical background of the proposed techniques for vibration analysis of structures.

\subsection{FFT analysis}

FFT is an optimized algorithm for performing the discrete Fourier transform (DFT), which is considered an efficient technique for analyzing stationary signals. DFT is defined by Eq. (1) (Proakis, 2006), where $x(n)$ is the discrete time signal, $N$ is the sampling period, $n$ and $k$ are the discrete time and frequency indexes, respectively, and the transformation kernel $W_{N}^{n k}$ is given by Eq. (2).

$$
\begin{aligned}
& X(k)=\frac{1}{N} \sum_{n=0}^{N-1} x(n) W_{N}^{n k} \text { for } 0 \leq k \leq N \text { and } 0 \leq n<N \\
& W_{N}^{n k}=\cos \left(\frac{2 n k \pi}{N}\right)+j \sin \left(\frac{2 n k \pi}{N}\right)
\end{aligned}
$$

\subsection{STFT analysis}

STFT is a technique capable of analyzing non-stationary signals. The basic idea of STFT is to apply FFT in sequence during short sampling periods. For each different time a different spectrum is obtained and the totality of such spectra indicates the time-frequency distribution (Gurley and Kareem, 1999).

STFT is defined by Eq. (3), where $x(t)$ and $h(t)$ are the signal to analyze and the window function, respectively, centered at time $\tau$.

$A_{t}(\omega)=\frac{1}{\sqrt{2 \pi}} \int_{-\infty}^{\infty} x(t) h(t-\tau) e^{-j \varpi \tau} d t$

The energy density spectrum at time $\tau$ is therefore

$P_{\mathrm{SP}}(t, \omega)=\left|A_{t}(\omega)\right|^{2}$

\subsection{DWT analysis}

DWT is considered an efficient tool for non-stationary signal analysis, providing significant characteristics of time and frequency (Chavez et al., 2010). DWT is described by Eq. (5), where $n$ represents the discrete time index, $x(n)$ is the discrete time original signal, $h(n)$ is the discrete time wavelet basis function, $N$ is the total number of $x(n)$ samples, $j$ is 
the time scaling, and $k$ is the shifting of the discrete wavelet function $h(n)$ through the input signal $x(n)$.

$\operatorname{DWT}_{j, k}=\sum_{N} x(n) \overline{h_{j, k}(n)}$

DWT is based on the Mallat algorithm (Mallat, 1999), which consists of high pass filter (HPF) and low pass filter (LPF) banks. According to the Mallat algorithm, the convolution of signal $x(n)$ with the LPF is known as the approximation level $\mathrm{L}\left(\mathrm{AC}_{\mathrm{L}}\right)$, and with the HPF is known as the detail level $\mathrm{L}\left(\mathrm{DC}_{\mathrm{L}}\right)$; the first decomposition of $x(n)$ is known as level 1 , and it is made of $\mathrm{DC}_{1}$ and $\mathrm{AC}_{1}$ that corresponds to the high-frequency and low-frequency components of the signal. The decomposition process continues on $\mathrm{AC}_{1}$, obtaining the level 2 made of $\mathrm{DC}_{2}$ and $\mathrm{AC}_{2}$ that corresponds to the high-frequency and low-frequency components of $\mathrm{AC}_{1}$, respectively. Once the discrete input signal $x(n)$ has been decomposed into the $L$ desired levels, the signal reconstruction is done by applying the decomposition process in an inverse way that is known as the inverse discrete wavelet transform (IDWT).

According to the DWT filter bank properties, the frequency band for an approximation $\mathrm{AC}_{\mathrm{L}}$ and a detail $\mathrm{DC}_{\mathrm{L}}$ is given by Eqs. (6) and (7), respectively, where $f_{\mathrm{s}}$ is the sampling frequency.

$\mathrm{AC}_{\mathrm{L}} \Rightarrow\left[0, \frac{f_{\mathrm{s}}}{2^{\mathrm{L}+1}}\right]$

$\mathrm{DC}_{\mathrm{L}} \Rightarrow\left[\frac{f_{\mathrm{s}}}{2^{\mathrm{L}+1}}, \frac{f_{\mathrm{s}}}{2^{\mathrm{L}}}\right]$

\subsection{WPT analysis}

WPT, like DWT, is a technique to decompose a signal repeatedly into successive low-frequency and high-frequency components. Yet, in WPT both the detail and the approximation coefficients are decomposed to create the full binary tree of band decomposition. The main advantage of using WPT over DWT is that it can provide uniform frequency bands; therefore, identifying more frequency components especially at high frequency is possible.

WPT is defined by Eq. (8), where $i, j$ and $k$ are the modulation, scale and translation parameters, respectively (Reda et al., 2006).

$\psi_{j, k}^{i}(t)=2^{j / 2} \psi^{i}\left(2^{j} t-k\right), \quad i=1,2,3, \ldots \ldots$

WPT is obtained through the next recursive equations, where $h(k), g(k)$ and $\psi^{i}$ are low-pass filter coefficients and highpass filter coefficients associated with the scaling function and the mother wavelet, respectively.

$$
\begin{aligned}
& \psi^{2 i}(t)=\sqrt{2} \sum_{k=-\infty}^{\infty} h(k) \psi^{i}(2 t-k) \\
& \psi^{2 i+1}(t)=\sqrt{2} \sum_{k=-\infty}^{\infty} g(k) \psi^{i}(2 t-k)
\end{aligned}
$$

\subsection{DWT/WPT combined with FFT analysis}

Two promising techniques that have not been employed to analyze civil structures are the combination of DWT with FFT, and WPT with FFT. These techniques have provided good results on analyzing motors (Amezquita-Sanchez et al., 2010) and CNC machine tools (Rangel-Magdaleno et al., 2010); nonetheless, these techniques can be useful tools to analyze civil structures, earthquakes, etc.

The combination of these analysis techniques allows observing the behavior of the signal throughout time and identifying a characteristic frequency related to the reconstruction of either DWT or WPT, without increasing the FFT frequency resolution (Rangel-Magdaleno et al., 2010). A specific frequency band of interest is isolated by means of DWT or WPT, and the FFT is applied on the reconstruction level to identify the characteristic frequencies within this band.

\section{Methodology}

This section shows the methodology followed for the development of the hardware-software system, as well as its characteristics and capabilities. The general system, software methodology, and control system methodology are described.

\subsection{Hardware-software system}

The schematic diagram of the hardware-software system is shown in Fig. 2, in which the implemented system consists of six units: earthquake simulator software (ESS), control system (CS), primary actuator (PA), primary sensor (PS), signal conditioner (SC), and data acquisition system (DAS). The ESS unit generates and modifies the signal that will be used to excite the structure. In addition, this unit allows analyzing either the generated signal or the acquired signal. The CS unit stores and reproduces the generated signal by ESS unit. The PA unit includes a commercial linear amplifier, which receives and conditions the generated signal by the CS, and an electrodynamic shaker, which is fed by the conditioned signal to excite the structure. The system uses multiple accelerometers as PS for measuring the acceleration simultaneously in one or several test points of the structure. Finally, the DAS unit acquires and stores all the measured signals by the sensors, conditioned and amplified by the SC unit; then, these signals are sent to the ESS unit for their analysis.

\subsection{ESS methodology}

The central part in the proposed methodology is the ESS unit. This unit is in charge of simultaneously generating the signal that will excite the structure and monitor it. EES has a graphical user interface (GUI) to load the data and to present the output. Depicted in Fig. 3, the ESS unit is divided into two main modules: simulator and analyzer modules. 


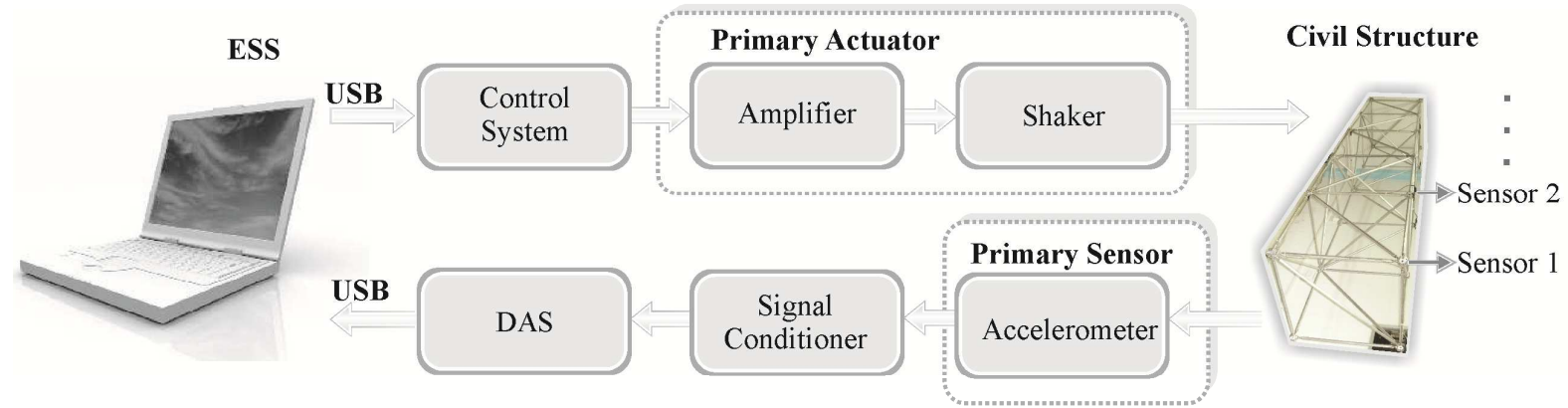

Fig. 2. Proposed methodology flow diagram.

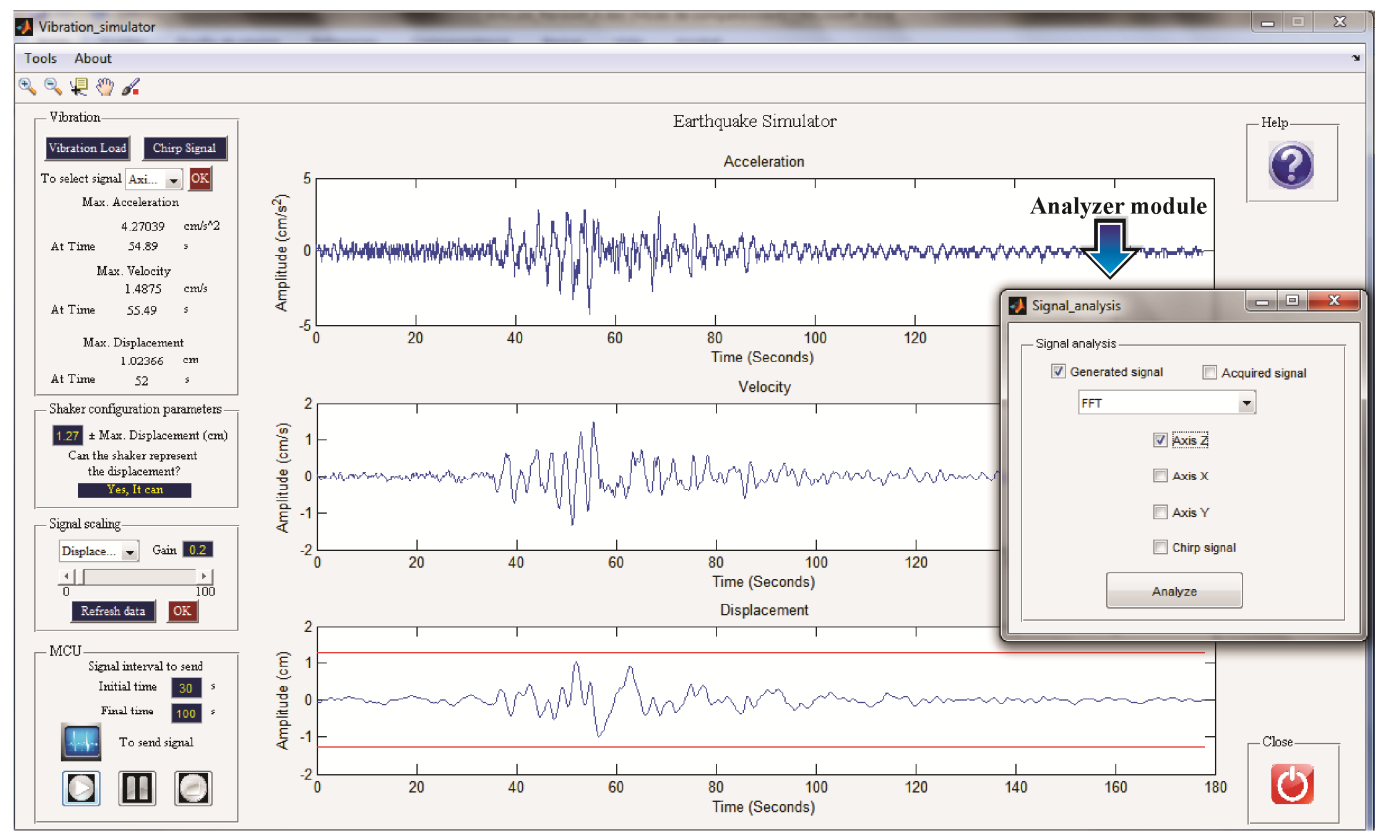

Fig. 3. ESS unit.

The simulator module is the main window, which appears when the program starts. The module is based on four stages: vibration, shaker configuration parameters, signal scaling, and master control unit (MCU). Figure 4 shows the overall process, depicted as follows:

a. Vibration. This stage generates or loads a signal that will be reproduced by the shaker. Earthquakes, synthetic signals, and ambient vibrations can be synthesized or generated by this stage. First, the push button "Vibration load" is used to load the data from either earthquake accelerations or ambient vibrations such as traffic, wind, etc. In addition, a synthetic signal with certain characteristics can be generated by the push button "Chirp Signal". Next, by means of a popup menu, the desired signal is selected. This popup menu was developed because in several cases the data loaded from a text file contains horizontal (axes $\mathrm{X}$ and $\mathrm{Y}$ ) and vertical (axis Z) accelerations. Then, the parameters of the shaker are set, this with the purpose of comparing the maximum displacement generated by the signal and the "max displacement" that can be represented by shaker. Finally, the push button "OK" is used to show the generated or loaded signal in the interface. Simultaneously, some characteristics of the signal such as "max acceleration", "max velocity" and "max displacement" are displayed (Fig. 4a).

b. Shaker configuration parameters. This stage sets the parameters to use any electrodynamic shaker (Fig. 4b). This stage is essential because it sets the signal that will feed the shaker according to displacement parameter of it. Moreover, this stage allows observing if the signal can be represented by the shaker. In all experimental 
(a)
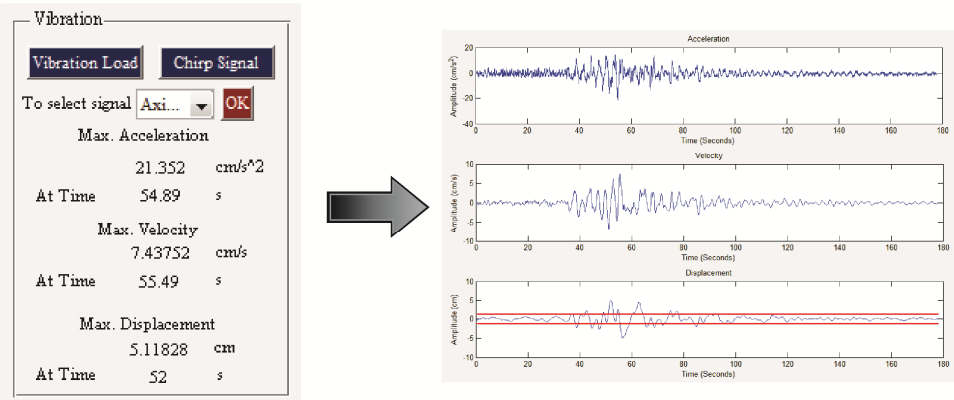

(b)
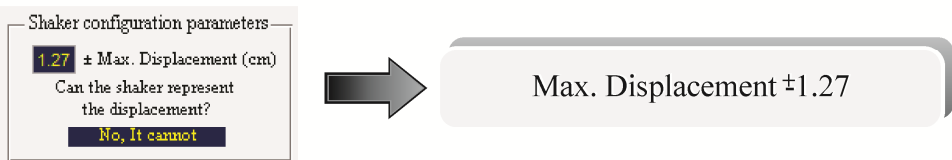

(c)
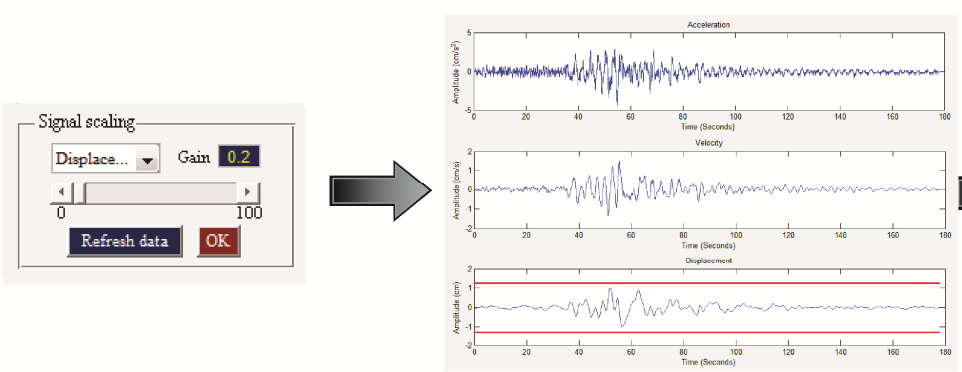

(f)
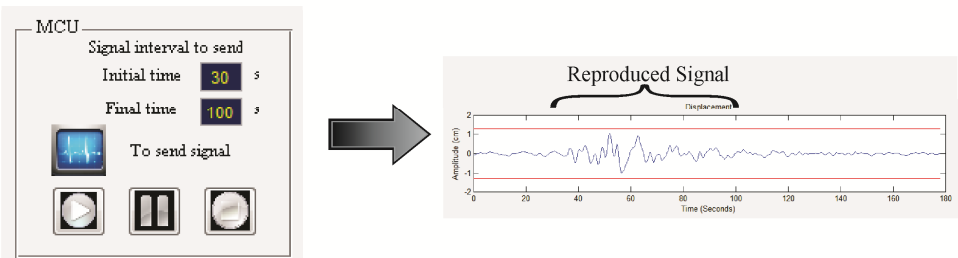

(d)

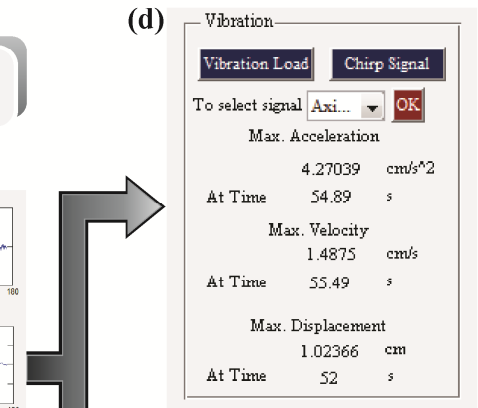

(e)

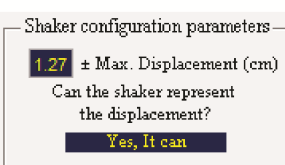

Fig. 4. Simulator module.

cases of this research, the displacement parameter was set to $\pm 1.27 \mathrm{~cm}$.

c. Signal scaling. This stage scales the signal in amplitude (Fig. 4c). First, the signal to be scaled is selected by means of a popup menu (acceleration, velocity or displacement). Next, a gain is established, which is multiplied by original signal to obtain the scaled signal. In order to display the scaled signal in the interface, it is necessary to press the push button "Refresh data". Simultaneously, "max acceleration", "max velocity" and "max displacement" are updated in the first stage (Vibration) (Fig. 4d). The Shaker configuration parameter (second stage) is updated (Fig. 4e) as well. This process is repeated until the user obtains the desired scaled signal. Finally, when the user observes a desired scaled signal, the push button "OK" has to be pressed to update the signal that will be sent to control system. d. MCU. This stage sends and controls the signal that will be reproduced by the shaker. First, the range of signal that will be reproduced is selected. After, when the user selects a desired region of the signal, the push button "To send signal" is used to send the signal to control system unit (Fig. 4f). In order to easily control the signal that will be reproduced by the control system unit, three push buttons "Play", "Pause", and "Stop" are used. The push button "Play" allows the reproduction of the generated signal in the interface. The push button "Pause" allows the user to pause the reproduction of the signal. Finally, the push button "Stop" allows the user to stop the reproduced signal.

The second module "Analyzer module", located in the menu "Tools", is used to analyze either the generated signal in the first module or the acquired signal by accelerometers through FFT, STFT, DWT, WPT, WPT-FFT and DWT-FFT analyses. Currently, an existing limitation in the commercial software for signal analysis by means of WPT is the difficulty in determining the frequency range of the reconstructed signal from 
various nodes (Zeng et al., 2008). Thus, it is necessary to determine the corresponding relationship between nodes of decomposition tree and frequency bands of signal subspace. A simple methodology to solve this problem was proposed by Zeng et al. (2008), which is implemented in the "Analyzer module". When the user chooses either WPT or WPT-FFT, the software automatically selects the appropriate node according to the chosen scale and frequency. The use of this module, by selecting WPT-FFT, is shown in Fig. 5. The selected options for this example in the "analyzer module" are: Earthquake of México 1985 as generated signal, WPT-FFT analysis, vertical acceleration (Axis Z), and 10 and $0.5 \mathrm{~Hz}$ for scale and frequency, respectively.

\subsection{Control system methodology}

The control system (CS) unit is based on the digital direct synthesis (DDS) technique (Romero-Troncoso and Espinosa-Flores, 1999). Depicted in Fig. 6, the CS unit is composed of five modules: communication interface (CI), dynamic memory, phase accumulator (PA), digital-analogconverter (DAC), and signal conditioner (SC). The CI receives the generated data from the EES unit by means of USB interface. Concurrently, the received data is stored in dynamic memories to form a look-up table (LUT). PA includes two stages, an adder and a register, which are in charge of reproducing the stored data to a desired frequency. Finally, the reproduced data is sent to DAC to generate the analog signal, which in turn is conditioned to feed the linear amplifier.

The output frequency of the phase accumulator is given by Eq. (10), in which $f_{\mathrm{o}}$ is the output frequency, $f_{\text {clk }}$ the clock frequency, $n$ the number of bits and $N$ varies from 0 to $2^{n-1}$ to set the desired frequency.

$f_{\mathrm{o}}=\frac{N}{2^{n}} f_{\mathrm{clk}}$

\section{Experimentation}

In this section, the experimental setup and the cases of study for validating the proposed system are presented.

\subsection{Experimental setup}

The experimental setup is shown in Fig. 7a; it consists of a five-bay space truss structure made of aluminum. Each assembled bay is a $707 \mathrm{~mm}$ cube-shaped structure. The test benches were performed using a Labworks Model ET-127 electrodynamic shaker as an excitation source, which can reproduce maximum $\pm 1.27 \mathrm{~cm}$ of displacement, powered by a Labworks Model PA-141 linear power amplifier. The amplifier is fed by a proprietary control system, which has a high-speed 14-bit digital-to-analog converter DAC2904 from Texas Instruments (Fig. 7b). Depicted in Fig. 7a, structural acceleration responses were measured using three triaxial accelerometers LIS3L02AS4 from STMicroelectronics mounted on top of first-bay (Sensor 1) third-bay (Sensor 2) and fifth-bay (Sensor 3), which aims at concurrently monitoring its behavior at different parts. The used accelerometers have a bandwidth of $750 \mathrm{~Hz}$, a user-selectable full scale of $\pm 2 \mathrm{~g} / \pm 6 \mathrm{~g}\left(\mathrm{~g}=9.81 \mathrm{~m} \mathrm{~s}^{-2}\right)$ and a $5 \times 10^{-4}$ resolution over a $100 \mathrm{~Hz}$ bandwidth. Accelerometer information is digitalized using 12-bit 4-channel ADS7841 ADC from Texas Instruments, with a maximum sampling rate of $200 \mathrm{kHz}$ for the four channels (Fig. 7c). Digital data acquisition was performed using a portable proprietary DAS; this system has eight input channels (Fig. 7b). The sampling frequency of the DAS system is set to $3.2 \mathrm{kHz}$. A proprietary Spartan 3E XC3S1600E FPGA platform running at $48 \mathrm{MHz}$ as the control system and DAS unit is used.

\subsection{Case studies}

The experimentation has three main objectives: firstly, to show the usefulness of ESS software to synthesize, generate and modify earthquake signals or any other excitation; secondly, to show that the system has the ability to concurrently monitor several test points of the structure; and thirdly, to show that the system is capable of analyzing the monitored signals by means of different techniques proposed in the EES software such as FFT, STFT, DWT, WPT, WPT-FFT and DWT-FFT.

In order to validate the effectiveness of the proposed hardware-software methodology, three experiments were performed. The first case of study focuses on the use of earthquakes signals. The next two cases of study allow observing the efficiency of the proposed system to generate or synthesize other types of signals such as ambient vibrations and synthetic waveforms. All experiments were carried out in a healthy truss structure.

In the first experiment, the earthquakes of México 1985 and Loma Prieta, California 1989, are used for exciting and analyzing the behavior of the structure during these excitations.

In the second experiment, an ambient vibration caused by traffic is used for exciting the structure. The used vibration was on-line monitored through two tri-axial accelerometers mounted onto a rigid bridge (Fig. 10a). The replication of these types of events in scaled structures is essential because it allows the user to observe the structural behavior during everyday situations.

Finally, in the last experiment, the efficiency of the system to generate a sinusoidal sweep with a frequency range of 22 to $26 \mathrm{~Hz}$ is displayed, aiming at finding the first natural frequency of the structure. 

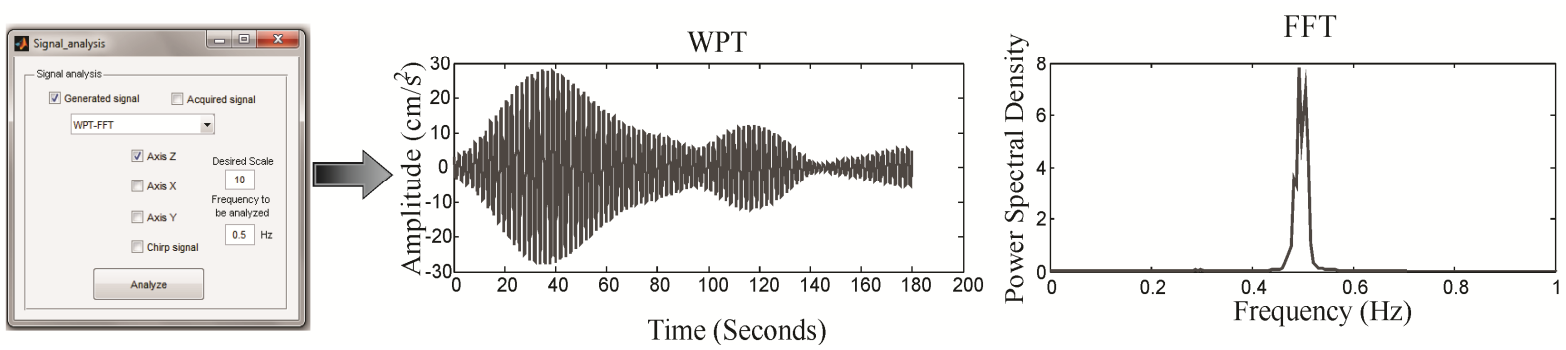

Fig. 5. Analyzer module.

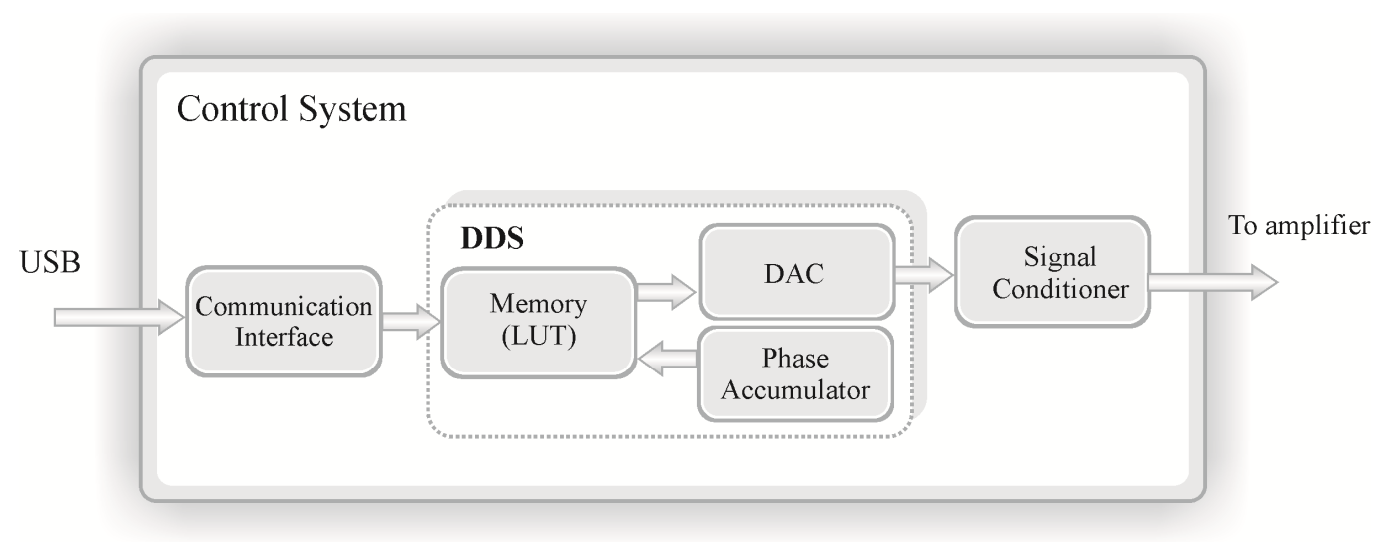

Fig. 6. Control system unit.

\section{Results}

In this section, the results of the proposed experimentation are presented. Also, the main advantages of the proposed system are discussed.

\subsection{Earthquakes}

Following the proposed methodology, the recorded acceleration of the earthquake in Mexico 1985 is loaded in the ESS (Fig. 8a). Then, the loaded signal is scaled to $0.97 \mathrm{~cm} \mathrm{~s}^{-2}$ maximum acceleration, $0.3836 \mathrm{~cm} \mathrm{~s}^{-1}$ maximum velocity and $0.18 \mathrm{~cm}$ maximum displacement. Additionally, the region with the maximum accelerations, 22 to $80 \mathrm{~s}$ (Fig. 8b), is selected to be reproduced by the shaker. The structural response of the first-, third- and fifth-bays are concurrently sensed (Fig. 8c-e). With the purpose to show the behavior of the structure during the excitation, FFT is selected for analyzing the measured vertical acceleration. Depicted in Fig. 8f$\mathrm{h}$, the measured time-domain signals are processed to obtain the FFT of the signals. The results show a static analysis of the structural response, where it is possible to identify the induced frequencies by the earthquake.

Alike the previous case, the recorded acceleration of the earthquake in Loma Prieta, California 1989, is loaded in the ESS (Fig. 9a). The loaded signal is scaled to $5.395 \mathrm{~cm} \mathrm{~s}^{-2}$ maximum acceleration, $0.7461 \mathrm{~cm} \mathrm{~s}^{-1}$ maximum velocity and $0.15 \mathrm{~cm}$ maximum displacement. Furthermore, the region with the maximum accelerations, 9 to $19 \mathrm{~s}$ (Fig. 9b), is selected to be reproduced by the shaker. As in the previous case, the structural response of the first-, third- and fifth-bays are concurrently sensed (Fig. 9c-e). STFT with a window of time duration $0.7 \mathrm{~s}$ is selected to analyze the measured vertical acceleration. Depicted in Fig. 9f-h, STFT allows observing the structural response throughout time, where a main frequency component, caused by the earthquake, is clearly observed.

In both case studies, the reproduced signal is compared with the experimental signal, demonstrating that the system is capable of exciting the structure with same characteristics of the generated signal by the ESS unit (Fig. 8c, f and Fig. 9a).

\subsection{Ambient vibration}

In the same way as in the previous test, the recorded acceleration produced by traffic on a bridge is loaded in the ESS (Fig. 10b). For this experiment, the signal is not scaled because it does not present long displacements. The loaded signal has the next characteristics: $52.2 \mathrm{~cm} \mathrm{~s}^{-2}$ maximum acceleration, $2.48 \mathrm{~cm} \mathrm{~s}^{-1}$ maximum velocity, and $0.15 \mathrm{~cm}$ maximum displacement. In addition, the reproduced signal is zoomed, where the maximum accelerations are from 505 to $525 \mathrm{~s}$. As in the previous test, the structural response of the 


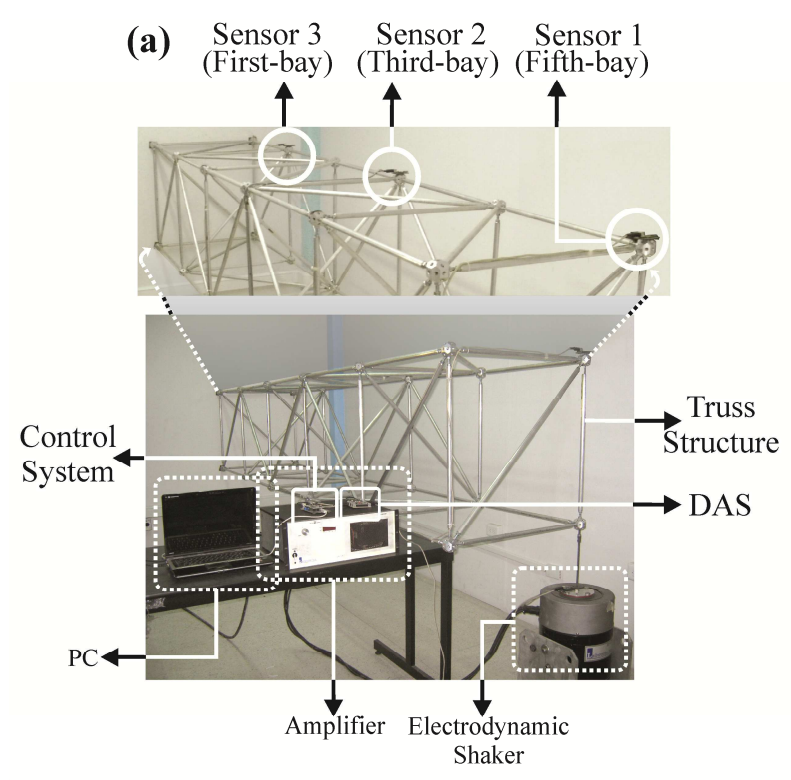

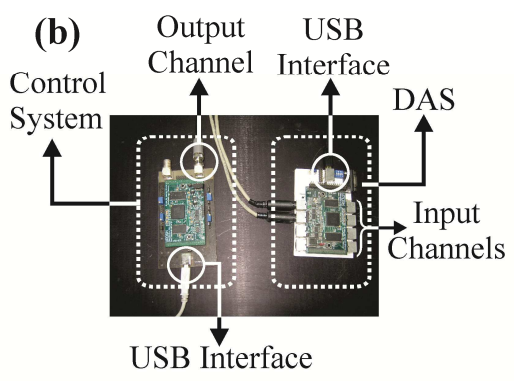

(c) Signal conditioning and

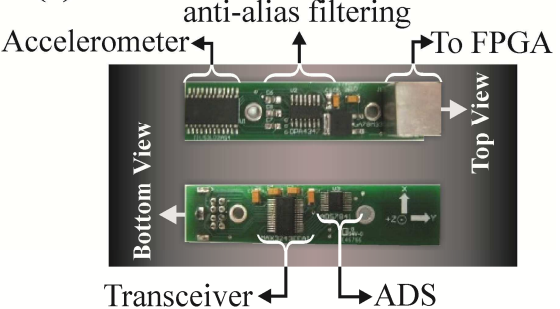

Fig. 7. Experimental setup.

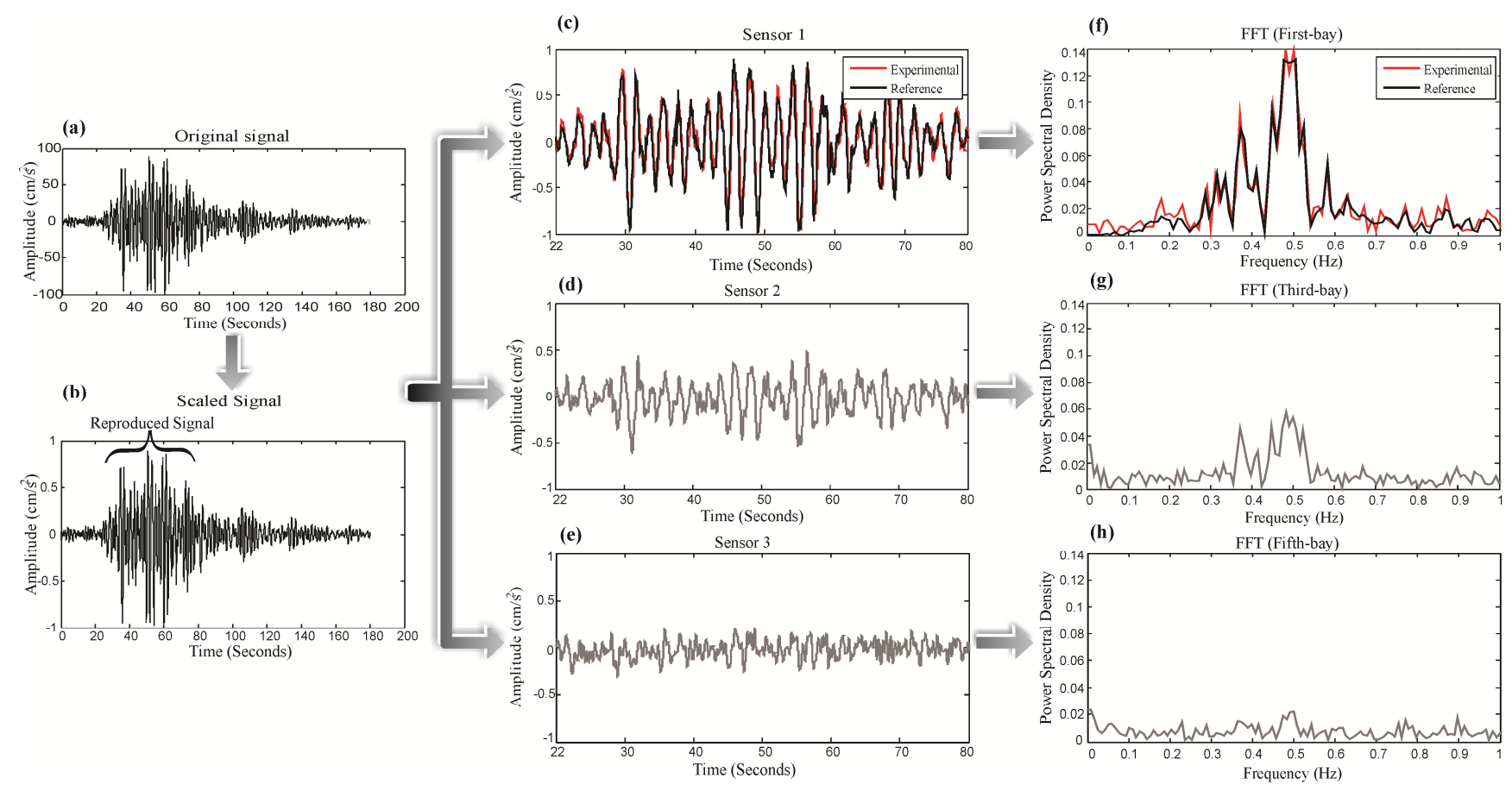

Fig. 8. Earthquake in México 1985.

first-, third- and fifth-bays are concurrently sensed (Fig. 10ce). DWT and WPT are selected for analyzing the monitored vertical accelerations. In addition, Daubechies (db4) is used as mother wavelet; due to that, $\mathrm{db} 4$ is the most suitable wavelet mother to decompose acceleration signals (Taha, 2010).
For this experiment, the region of interest lies below $3 \mathrm{~Hz}$, owing to the fact that the caused frequencies by traffic are low; therefore, the recommended decomposition level for DWT is DC10 with an active frequency band from 1.56 to $3.25 \mathrm{~Hz}$, and the recommended node for WPT is [10 1] with an active frequency band from 1.5625 to $3.125 \mathrm{~Hz}$. Depicted in Fig. 10f-k, DWT and WPT are applied to the time-domain 


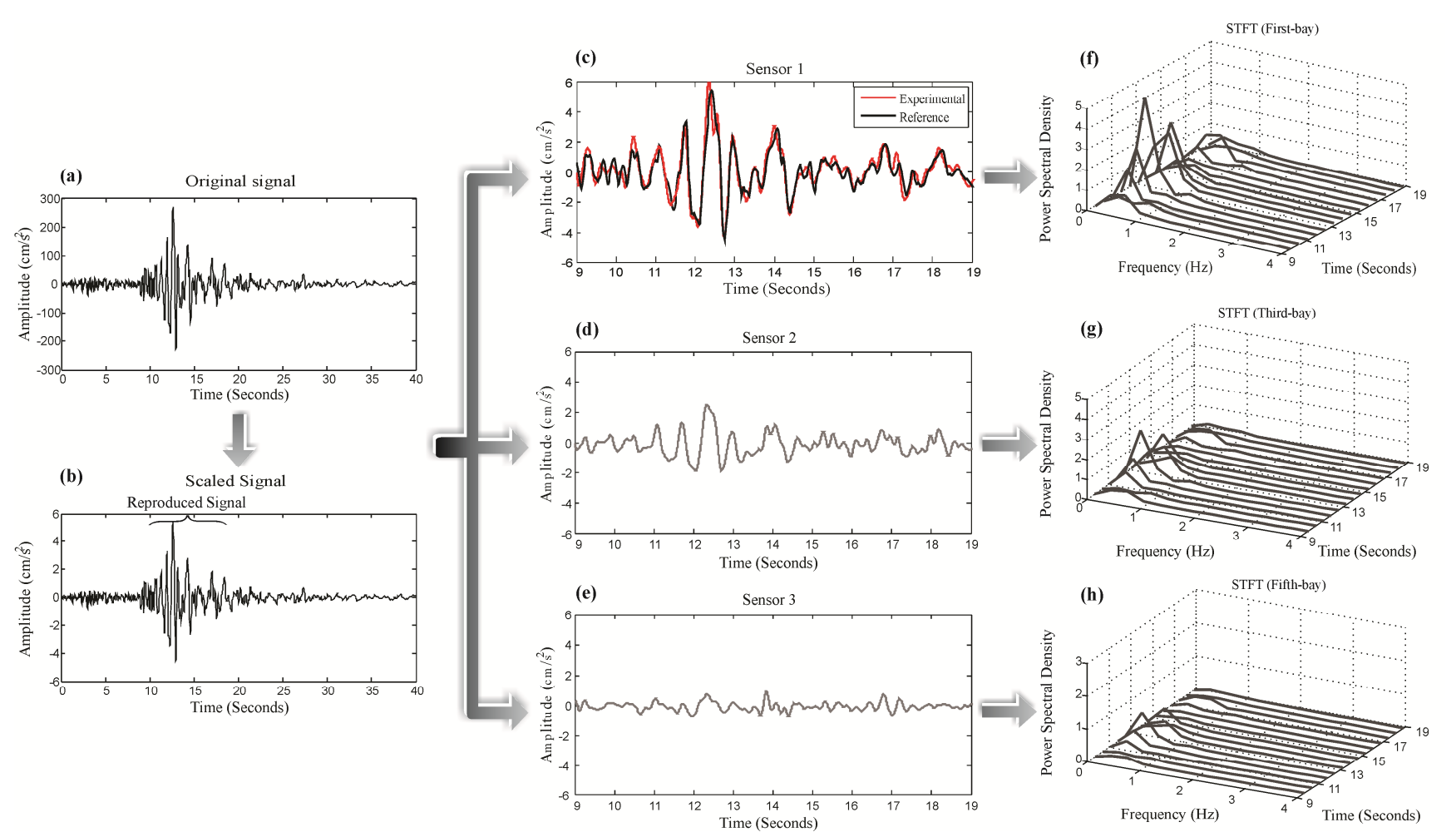

Fig. 9. Earthquake in Loma Prieta, California 1989.

signal to obtain the decomposition and reconstruction of the signal. The reconstructed wavelet signals allow observing the structural response throughout time, where the caused frequencies by traffic are clearly identified in this frequency band. Like in the previous test, the reproduced signal is compared with the experimental signal (Fig. 10c), where it is possible to observe that the system is capable of reproducing the signal generated by the ESS unit with great accuracy.

\subsection{Synthetic signal}

Unlike the two previous experiments, the tool "Chirp signal" was used to generate the exciter signal. The generated signal has the following characteristics: $10 \mathrm{KHz}$ sampling frequency, $10 \mathrm{~s}$ running time for the experiment, $3900 \mathrm{~cm} \mathrm{~s}^{-2}$ maximum acceleration, $20.72 \mathrm{~cm} \mathrm{~s}^{-1}$ maximum velocity and $0.11 \mathrm{~cm}$ maximum displacement; therefore, the scaling is not necessary. Like the previous test, the structural response of the first-, third- and fifth-bays are concurrently sensed (Fig. 11b-d). To analyze the behavior of the structure, WPTFFT and DWT-FFT are selected for analyzing the measured vertical acceleration. Similar to previous experiment, $\mathrm{db} 4$ is used as mother wavelet in both DWT-FFT and WPT-FFT analysis.

For this experiment, the region of interest lies below $26 \mathrm{~Hz}$; therefore, the recommended decomposition level for DWT is DC7 with an active frequency band from 12.5 to
$25 \mathrm{~Hz}$, and for WPT is [9 4] with an active frequency band from 21.875 to $25 \mathrm{~Hz}$. Once the decomposition level for DWT and the node for WPT have been established, the timedomain signal is processed to obtain the decomposition and reconstruction of the signal, which are shown in Fig. 11e-j. In both cases, the result wavelet contains the time evolution of the natural frequency of the structure, and to locate this frequency, the FFT is applied to obtain the spectrum, where a natural frequency is clearly appreciated (Fig. $11 \mathrm{k}-\mathrm{p}$ ).

\subsection{Methodology comparative}

In order to validate the main advantages of the proposed system to other similar used systems, a comparison of the main features is shown in Table 1.

Of the works shown in Table 1, only Dominguez et al. (2007) used proprietary software, while the others used commercial software. Dominguez et al. (2007) reported the use of a system capable of exciting a structure under several types of signals, in addition to monitoring several test points concurrently in order to analyze the behavior of a truss structure. On the other hand, Reynolds and Pavic (2002), Zivanović et al. (2006) and Ibrahim and Reynolds (2008) reported the use of a portable system capable of simultaneously exciting, monitoring and analyzing a structure. However, all these works present several disadvantages. Firstly, the used systems are unable to analyze the monitored signals 


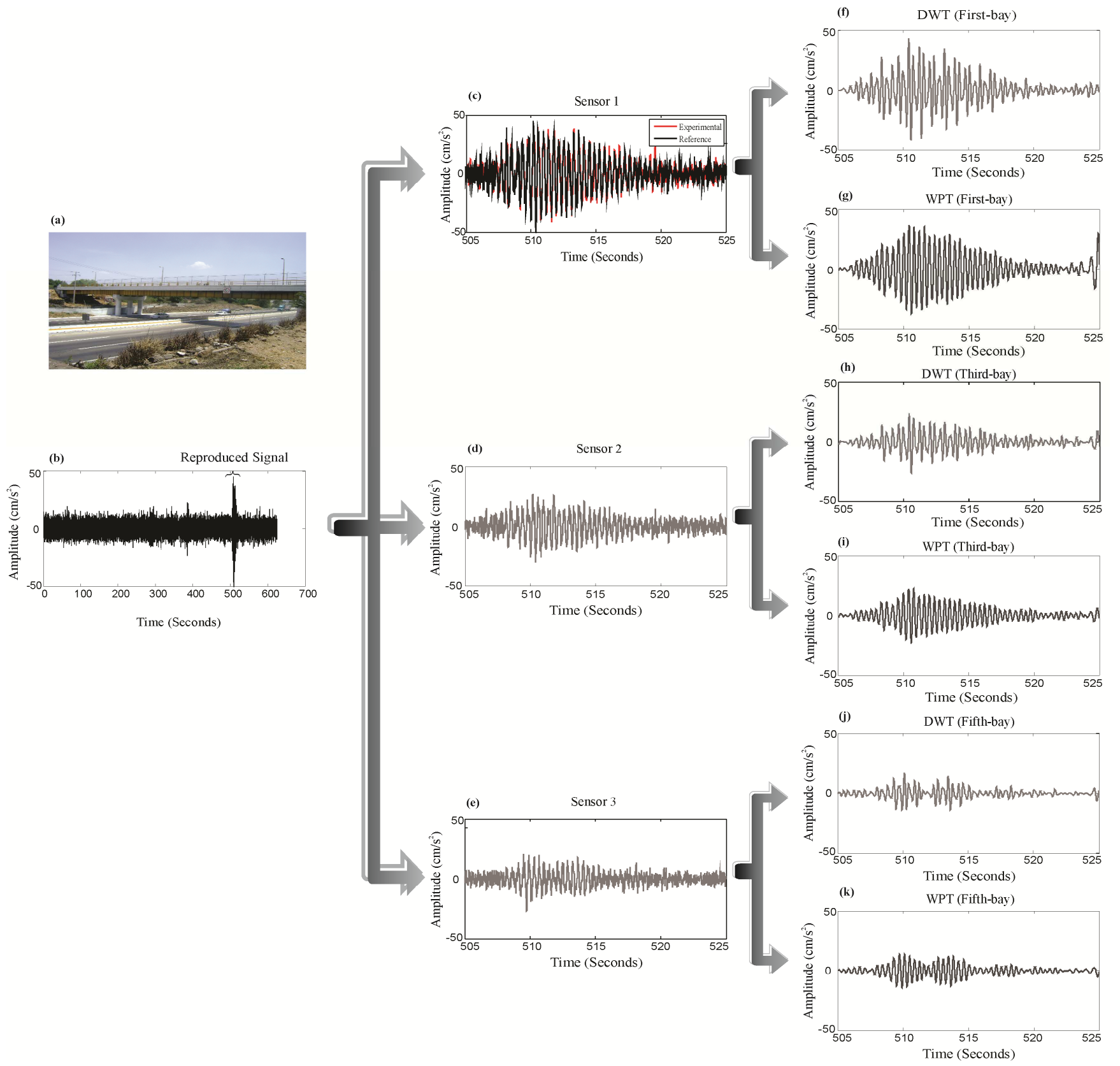

Fig. 10. Ambient vibration.

by means of different analysis techniques. In addition, they do not allow introducing or synthesizing a signal; thus, earthquake signals and ambient vibrations cannot be reproduced (Reynolds and Pavic, 2002; Zivanović et al., 2006 and Ibrahim and Reynolds, 2008). Secondly, the monitoring of several test points concurrently is not performed (Zivanović et al., 2006). Third, the use of two software programs, one for generating the signal that excites the structure and another for the analysis of the measured signal, means portability of the system is not possible (Dominguez et al., 2007).
In this work, three experiments demonstrate that the proposed single system is capable of either generating or synthesizing, and both modifying and analyzing, several types of signals as well as monitoring several test points concurrently to evaluate the behavior of the structure by means of several analysis techniques, being also a portable system. 


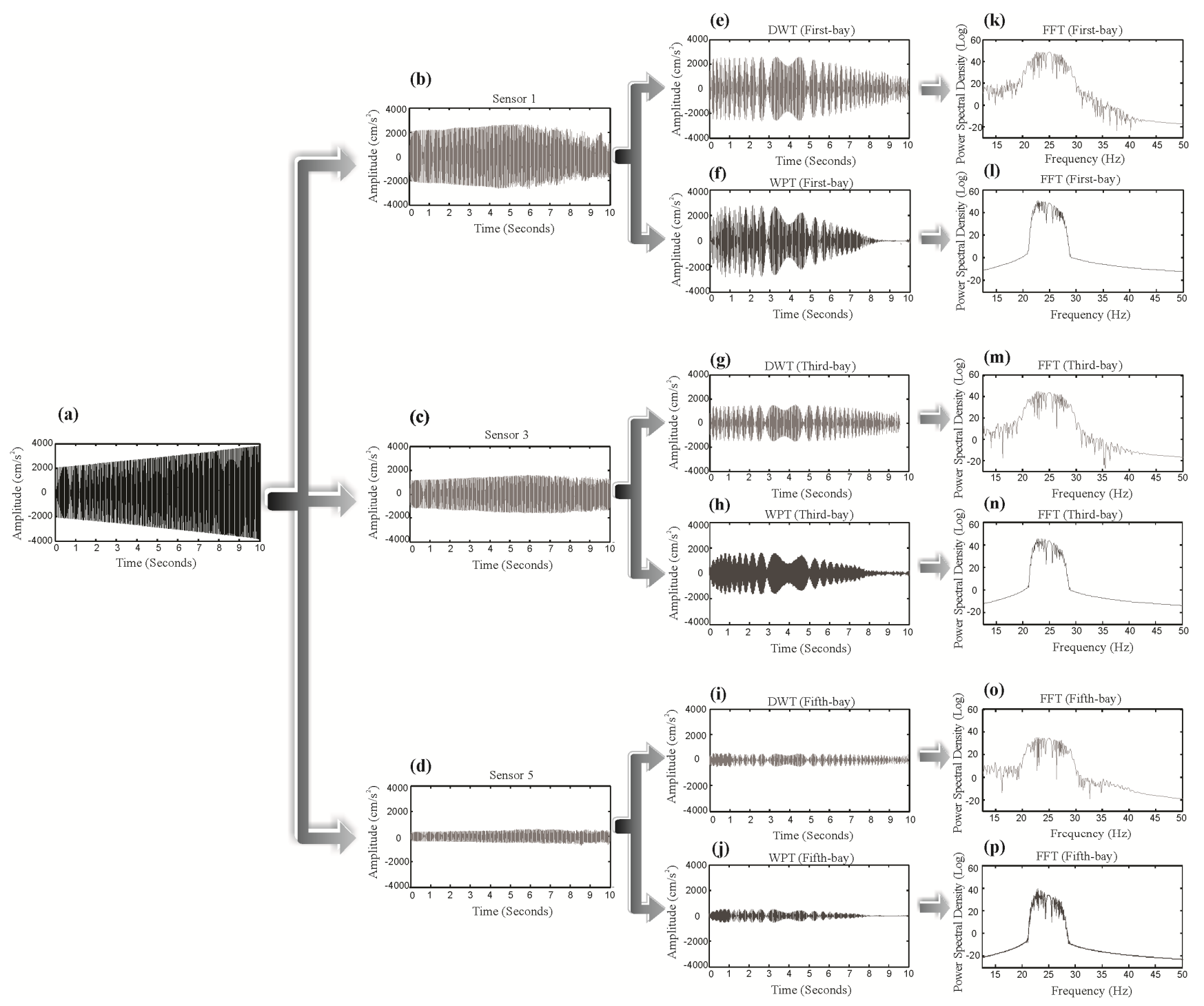

Fig. 11. Synthetic signal.

Table 1. Features comparison between the proposal and reported works.

\begin{tabular}{llllllc}
\hline Work & $\begin{array}{l}\text { Generate } \\
\text { multiple } \\
\text { signals }\end{array}$ & $\begin{array}{l}\text { Modify } \\
\text { signals }\end{array}$ & $\begin{array}{l}\text { Monitor } \\
\text { multiple test } \\
\text { points }\end{array}$ & $\begin{array}{l}\text { Multiple } \\
\text { techniques of } \\
\text { analyzing }\end{array}$ & $\begin{array}{l}\text { One system for } \\
\text { generating, monitoring } \\
\text { and analyzing }\end{array}$ & $\begin{array}{l}\text { Portability } \\
\text { Reynolds and Pavic (2002) }\end{array}$ \\
YES & NO & YES & NO & YES & YES \\
Zivanović et al. (2006) & YES & NO & NO & NO & YES & YES \\
Dominguez et al. (2007) & YES & NO & YES & NO & NO & NO \\
Ibrahim and Reynolds (2008) & YES & NO & YES & NO & YES & YES \\
This work & YES & YES & YES & YES & YES & YES \\
\hline
\end{tabular}




\section{Conclusions}

Earthquakes are seismic waves that cause the greatest catastrophes: human, economic and structural. Therefore, it is necessary to have a system capable of analyzing, monitoring and reproducing them. Seismic hazard analysis is one of the most important processes for earthquake safety assessment of structures and the designing of earthquake resistant structures.

This paper proposes a hardware-software system for exciting, monitoring and analyzing simultaneously a structure under earthquakes, ambient vibrations and synthetic signals in real-time. The proposed system has different tools to generate and to modify the different signals either generated or acquired, as well as their analysis by means of several analysis techniques such as FFT, STFT, DWT, WPT, DWT-FFT and WPT-FFT. Three experiments have been presented in this work. In the first experiment, the system proves to be a useful tool to synthesize, modify and reproduce real earthquake signals with great accuracy, which is fundamental in the analysis and design of structures. The second and the third experiment are carried out to show the efficiency of the system to generate or to synthesize other types of signals such as ambient vibration and synthetic signals, aiming at knowing other characteristics like natural frequencies.

The proposed system is a useful tool in the analysis of earthquake effects on structures in order to help the improvement of its performance, resistance, design, and life service. In addition, it has tools to generate other types of signals for calculating other structure characteristics, showcased in the developed experiments. Furthermore, the feature comparison between the proposed system and other reported works highlights the advantages of using the proposed system.

Acknowledgements. This project was partially supported by CONACyT scholarship 213849 and SEP-CONACyT 84723 project.

Edited by: M. E. Contadakis

Reviewed by: A. Garcia-Perez and another anonymous referee

\section{References}

Amezquita-Sanchez, J. P., Cabal-Yepez, E., Romero-Troncoso, R. J., Osornio-Rios, R. A., and Garcia-Perez, A.: Determination of system frequencies in mechanical systems during shutdown transient, J. Sci. Ind. Res., 69, 415-421, 2010.

Brownjohn, J. M. W., Moyo, P., Omenzetter, P., and Lu, Y.: Assessment of highway bridge upgrading by dynamic testing and finite-element model updating, J. Bridge Eng., 8, 162-172, 2003.

Chavez, O., Millan-Almaraz, J. R., Pérez-Enríquez, R., ArzateFlores, J. A., Kotsarenko, A., Cruz-Abeyro, J. A., and Rojas, E.: Detection of ULF geomagnetic signals associated with seismic events in Central Mexico using Discrete Wavelet Transform, Nat. Hazards Earth Syst. Sci., 10, 2557-2564, doi:10.5194/nhess-102557-2010, 2010.
Chen, B., Xu, Y. L., and Qu, W. L.: Evaluation of atmospheric corrosion damage to steel space structures in coastal areas, Int. J. Solids Struct., 42, 4673-4694, 2005.

Dolce, M. and Cardone, D.: Theoretical and experimental studies for the application of shape memory alloys in civil engineering, J. Eng. Mater. Technol., 128, 302-311, 2006.

Dominguez, A., Sedaghati, R., and Stiharu, I.: Modeling and application of MR dampers in semi-adaptive structures, Comput. Struct., 86, 407-415, 2007.

Dyke, S. J.: Design and development of the Washington University seismic simulator facility, in: Proc. 12th Eng. Mech, ASCE, San Diego, 762-765, 1998.

Ersubasi, F. and Korkmaz, H. H.: Shaking table tests on strengthening of masonry structures against earthquake hazard, Nat. Hazards Earth Syst. Sci., 10, 1209-1220, doi:10.5194/nhess-101209-2010, 2010.

Gurley, K. and Kareem, A.: Applications of wavelet transforms in earthquake, wind and ocean engineering, Eng. Struct., 21, 149167, 1999.

Han, J. G., Wei-Xin Ren, W. X., and Sun, Z. S.: Wavelet packet based damage identification of beam structures, Int. J. Solids Struct., 42, 6610-6627, 2005.

Ibrahim, Z. and Reynolds, P.: Modal testing of a cantilever grandstand, in: International conference on construction and building technology (ICCBT2008), 16-20, Kuala Lumpur, Malaysia, 2008.

Jeyasehara, C. A., Kumarb, K. S., Muthumanib, K., and Lakshmananb, N.: Seismic performance evaluation methodologies for civil engineering structures, Indian J. Eng. Mater. Sci., 16, 220 228, 2009.

Johnson, R., Padgett, J. E., Maragakis, M. E., DesRoches, R., and Saiidi, M. S.: Large scale testing of nitinol shape memory alloy devices for retrofitting of bridges, Smart Mater. Struct., 17, 1-10, 2008.

Kamanli, M. and Balik, F. S.: The behaviour of roof gable walls under the effect of earthquake load, Nat. Hazards Earth Syst. Sci., 10, 251-263, doi:10.5194/nhess-10-251-2010, 2010

Mallat, S.: A Wavelet tour of signal processing, 2nd Edn., Academic Press, 1999.

Nagarajaiah, S.: Adaptive passive, semiactive, smart tuned mass dampers: identification and control using empirical mode decomposition, hilbert transform, and short-term fourier transform, Struct Contr Health Monit., 16, 800-841, 2009.

Nagarajaiah, S. and Basu, B.: Output only modal identification and structural damage detection using time frequency and wavelet techniques, Earthq. Eng. Eng. Vib., 8, 583-605, 2009.

Nakata, N.: Acceleration trajectory tracking control for earthquake simulators, Eng. Struct., 32, 2229-2236, 2010.

Ovanesova, A. V. and Suárez, L. E.: Applications of wavelet transforms to damage detection in frame structures, Eng. Struct., 26, 39-49, 2004

Proakis, J. G. and Manolakis, D. K.: Digital Signal Processing, Principles and Applications, Fourth Edition, Prentice-Hall, New Jersey, USA, 2006.

Rangel-Magdaleno, J. J., Romero-Troncoso, R. J., Osornio-Rios, R.A., Cabal-Yepez, E., and Dominguez-Gonzalez, A.: FPGAbased vibration analyzer for continuous CNC machinery monitoring with fused FFT-DWT signal processing, IEEE Trans. Instrum. Meas., 59, 3184-3194, 2010 
Reda, M. M., Noureldin, A., Lucero, J. L., and Baca, T. J.: Wavelet transform for structural health monitoring: A compendium of uses and features, Struct. Health. Monit., 5, 267-295, 2006.

Ren, W. X., Tong Zhao, T., and Harik, I. E.: Experimental and analytical modal analysis of steel arch bridge, J. Struct. Eng., 130, 1022-1031, 2004.

Ren, W. X., Peng, X. L., and Lin, Y. Q.: Experimental and analytical studies on dynamic characteristics of a large span cable-stayed bridge, Eng. Struct., 27, 535-548, 2005.

Reynolds, P. and Pavic, A.: Modal testing of a sport stadium, in: Proc. of the 20th. International Modal Analysis Conference (IMAC XX), Los Angeles, California, USA, 1037-1043, 2002.

Romero-Troncoso, R. J. and Espinosa-Flores, G.: Phase accumulator synthesis algorithm for DDS applications, Electron. Lett., 35, 770-772, 1999.

San Bartolome, A., Quiun, D., and Zegarra, L.: Effective system for seismic reinforcement of adobe houses, in: 13th World conference on Earthquake Engineering, 3321, Vancouver, B.C., Canada, 2004.
Sun, Z. and Chang, C. C.: Structural damage assessment based on wavelet packet transform, J. Struct. Eng., 128, 1354-1361, 2002.

Taha, M. M. R.: A neural-wavelet technique for damage identification in the ASCE benchmark structure using phase II experimental data, Adv. Civ. Eng., doi:10.1155/2010/675927, 2010.

Van de Lindt, J. W. and Potts, A.: Shake table testing of a Superelastic Shape Memory Alloy response modification device in a wood shearwall, J. Struct. Eng., 134, 1343-1352, 2008.

Zeng, X., Zhao, W., and Sheng, J.: Corresponding relationships between nodes of decomposition tree of wavelet packet and frequency bands of signal subspace, Acta Seismol. Sin., 21, 91-97, 2008.

Zivanović, S., Pavic, A., and Reynolds, P.: Modal testing and FE model tuning of a lively footbridge structure, Eng. Struct., 28, 857-868, 2006. 